\title{
Experimental and Computational Analysis of Microbial Inactivation in a Solid by Ohmic Heating Using Pulsed Electric Fields
}

\author{
M. Á. Ariza-Gracia ${ }^{\mathrm{a}, \mathrm{b}}$, M. P. Cabello ${ }^{\mathrm{a}}$, G. Cebrián ${ }^{\mathrm{c}}$, B. Calvo ${ }^{\mathrm{a}, \mathrm{d}}$, I. Álvarez ${ }^{\mathrm{c}}$ \\ ${ }^{a}$ Aragón Institute for Engineering Research (I3A), University of Zaragoza, Zaragoza, Spain. \\ ${ }^{b}$ ARTORG Center for Biomedical Engineering Research, University of Bern, Switzerland. \\ ${ }^{c}$ Departamento de Producción Animal y Ciencia de los Alimentos. Tecnología de los Alimentos. Facultad de Veterinaria. \\ Instituto Agroalimentario de Aragón (IA2). Universidad de Zaragoza, Zaragoza, Spain. \\ ${ }^{d}$ Centro de Investigación Biomédica en Red en Bioingeniería, Biomateriales y Nanomedicina (CIBER-BBN), Spain.
}

\begin{abstract}
Pulsed electric field technology (PEF) has traditionally been used as a technique to inactivate microorganisms in liquid foods at temperatures below those used in heat treatments; however, application of high-intensity PEF (E >1 $\mathrm{kV} / \mathrm{cm})$ at high frequencies $(>10 \mathrm{~Hz})$ can allow rapid and volumetric solid food electrical heating in order to replace traditional convection/conduction heating that progresses from the heating medium to the inside of the product. This investigation is the first one to evaluate the inactivation of Salmonella Typhimurium 878 in a solid product (cylinder of technical agar used as reference solid) by applying PEF treatments $(2.5$ and $3.75 \mathrm{kV} / \mathrm{cm}$, and up to 9,000 microseconds) at $50 \mathrm{~Hz}$. The evolution of temperature in different locations of the agar cylinder was measured by observing the variability of heating rates depending on location and PEF intensity. Microbial inactivation was determined and compared with isothermal heat treatments that predicted similar inactivation values, but did not detect additional inactivation. Computational analysis enabled us to predict temperature and microbial inactivation for any spatial and temporal distribution of the cylinder agar by detecting the coldest point in the transition zone between the high-voltage electrode, the agar, and the plastic container of the treatment chamber. In order to evaluate the variability of the temperature, computational predictions were done each $0.5-\mathrm{mm}$. The difference between the coldest and the hottest point (e.g. at the center of the cylinder) resulted in around $10{ }^{\circ} \mathrm{C}$ and 10 seconds variation in temperature and processing time, respectively. In any case, it was possible to obtain 5 - $\log _{10}$-reductions after 60 seconds of PEF treatments when using $2.5 \mathrm{kV} / \mathrm{cm}$ and $50 \%$ reduction for $3.75 \mathrm{kV} / \mathrm{cm}$. These results suggested the potential of PEF technology as a rapid heating system based on ohmic heating for microbial inactivation in solid food products.
\end{abstract}


Keywords: Pulsed electric fields, Microbial inactivation, Numerical simulation, Ohmic heating, Temperature

\section{1. Introduction}

Pulsed electric fields (PEF) technology has been extensively investigated, and is currently being applied by the food industry as an alternative to the thermal pasteurization of liquid products, mainly fruit juices. Thanks to the electropermeabilizing effect of PEF, vegetative cells of pathogenic and spoilage microorganisms can be inactivated using lower temperatures than those applied in thermal treatments, thereby permitting the food industry to maintain fresh-like characteristics of foods (Buckow, Ng, \& Toepfl, 2013). PEF treatment applies electric fields of high intensity $(>0.1 \mathrm{kV} / \mathrm{cm})$ and short duration (from milliseconds to microseconds) to a product placed between two electrodes with only a minimal increase in product temperature, or at least, with an aim to minimize thermal effects (Barbosa-Canovas, Fernandez-Molina, \& Swanson, 2001). However the application of a voltage between two electrodes separated by a certain distance (electric field) within which the product to be treated by PEF is placed leads to the passage of an electrical current that induces ohmic heating of the product by Joule effect. This heating effect $(W)$ is defined according to the following equation:

$$
W=\int_{0}^{\omega} \sigma \mathbf{E}^{2} d t
$$

where $\sigma$ is the electrical conductivity of the treated medium or product $(\mathrm{S} / \mathrm{m}), \mathbf{E}$ is the electric field strength $(\mathrm{V} / \mathrm{m})$; and $d t$ is the time (s) during which the field strength is applied (Sastry \& Li, 1996).

This indicates that any increment in these parameters (including pulse width and frequency, considered within the time parameter) increases the energy transferred to the treated medium. In the case of field strength, slight modifications square transferred energy. This fact opens up new possibilities for the use of PEF as a highcapacity heating system, thereby improving the possibilities of ohmic heating based on rapid and relatively uniform heating inside the food, similarly to microwaves or radio frequency, but with a higher penetration capacity in the product, and attaining an energetic efficiency close to $90 \%$ (Sastry, 2004). Moreover, the electropermeabilizing effect of PEF would allow for an increase in the electrical conductivity of the product, thereby augmenting energy transfer while pulsing, and enabling a greater uniformity along the whole product, particularly if solid products are treated. For this purpose, moderate electric fields (MEF) using electric field 
strengths up to $1 \mathrm{kV} / \mathrm{cm}$ are under research (Sastry, 2008, Kaur \& Singh, 2016), as well as the limitation of electrochemical reactions associated with the traditional application of ohmic heating (Samaranayake, Sastry, \& Zhang, 2005).

Recently, Timmermans et al. (2019) showed the microbial lethal effect of moderate-intensity Pulsed Electric Fields by applying electric field strengths up to $5 \mathrm{kV} / \mathrm{cm}$ and $81^{\circ} \mathrm{C}$ to different fruit juices and by comparing the effect to that of PEF at higher field strengths and to that of traditional heat treatments, respectively. The application of moderate PEF intensities has shown less dependence on the $\mathrm{pH}$ of the treatment medium and on the treated microbial species than PEF or heat, thus facilitating the implementation of moderate PEF on an industrial level. Combined with the heating capacity of MEF applied to solid products, these benefits can represent a new strategy to overcome the limitation of traditional heating of solid products, which results in heterogeneous heat treatments, thereby affecting quality but, more importantly, reducing the uniformity of microbial lethal effectiveness and thereby generating a risk for food safety (Yildiz-Turp, Sengun, Kendirci, \& Icier, 2013). Due to this, although ohmic heating and MEF can generate a relatively uniform heating effect, this is a key point to be evaluated when applying heat treatments independently of the system. When ohmic heating, MEF, and PEF are applied, temperature-dependent food properties such as electrical conductivity, density, viscosity and thermal conductivity are modified. These changes may exert an influence on electric field distribution and heating, thus compromising the treatment's uniformity and thereby the most important criterion for a successful technology, which is safety. Thus, it is necessary to measure potential variation. However, it is difficult to achieve experimental information regarding the distribution of electric field strength and temperature in the treated product (Saldaña, Puertolas, Condon, Alvarez, \& Raso, 2010).

Numerical simulation can provide detailed knowledge of the temporal and spatial distribution of the food's electric field strength and temperature in the treatment chamber. With this information, an approach designed to estimate the degree of microbial inactivation in an inhomogeneous MEF or PEF process can be implemented if the heat resistance of the microorganisms of interest under isothermal conditions is known. Numerical simulation has proven to be a very useful tool to optimize treatment chambers in ohmic, MEF, and PEF treatments (Gerlach, Alleborn, Baars, Delgado, Moritz, \& Knorr, 2008, Wölken, Sailer, Maldonado-Parra, Horneber, \& Rauh, 2017, Shim, Lee, \& Jun, 2010, McLaren, Kopatz, Smith, \& Jain, 2019). Research on the 
microbial lethality of moderate-intensity PEF in solids due to Joule effect is nevertheless scarce. Moreover, only a limited amount of information is available regarding the validation thereof by numerical simulation tools that study the uniformity of both temperature and field strength in the treatment chamber, and regarding the optimization of treatment conditions designed to achieve a certain level of inactivation of the pathogenic microorganisms of interest. That is the objective of this investigation, which can serve as a starting point to evaluate PEF technology as a further new system that can be applied to achieve rapid pasteurization of solid products.

\section{Materials and methods}

The present study is two-fold. On the one hand, it was carried out a first set of PEF experiments on a solid agar cylinder in order to evaluate the thermal effect of different field strengths on heating rates at different distinct points within the solid, and on the inactivation of Salmonella Typhimurium 878. On the other hand, a numerical model (Finite Element Model-FEM) was applied in order to predict the degree of ohmic heating $(\mathrm{OH})$ in the solid, thereby estimating the microbial inactivation of Salmonella Typhimurium 878 and evaluating the uniformity of its lethality in the agar cylinder with the purpose of reducing the number of experiments in laboratory. In order to estimate the degree of microbial inactivation, microbial thermal resistance at isothermal conditions was determined.

\subsection{PEF system}

The PEF equipment used in this investigation was supplied by ScandiNova (Modulator PG, ScandiNova, Uppsala, Sweden). The device generates square wave pulses of a width of $3 \mu s$ with frequencies varying from 0.5 to $300 \mathrm{~Hz}$. The maximum output voltage and current are limited to $30 \mathrm{kV}$ and 200 A, respectively. The equipment consists of a direct current power supply which converts the 3 -phase line voltage to a regulated DC voltage. It charges up to six IGBT switching modules (high-power solid-state switches) to a primary voltage around $1000 \mathrm{~V}$. An external trigger pulse gates all the modules and controls their discharge to a primary pulsed signal of around $1000 \mathrm{~V}$. Finally, a pulse transformer converts the primary $1000 \mathrm{~V}$ pulse to a 3- $\mu$ s high-voltage pulse of desired high voltage. For safe manipulation of the PEF device, and in order to obtain rectangular 3- $\mu$ s pulses, the electric current delivered in the treatment chamber has to lie within a range of 80 to $150 \mathrm{~A}$. 
The treatment chamber consists of a cylindrical Teflon (polytetrafluoroethylene) tube closed with two polished stainless steel cylinders of 20-mm diameter and 2-mm thickness, and separated 20-mm from each other where the agar cylinder of 20-mm height and 20-mm diameter is located (Figure 1). To avoid movement of the electrodes due to pressure when increasing temperature, two Teflon caps were screwed to the Teflon cylinder. Three holes of 2-mm diameter in the cylindrical Teflon tube were used to introduce the temperature probes to register the temperature at different locations of the agar cylinder. Actual voltage and current intensity applied in the treatment chamber were measured with a high voltage probe (Tektronix, P6015A, Wilsonville, Oregon, USA) and a current probe (Stangenes Industries Inc. Palo Alto, California, USA), respectively, connected to an oscilloscope (Tektronix, TDS 220, Wilsonville, Oregon, USA)

\subsection{PEF heating curves in agar}

For benchmark, technical agar (Oxoid Basingstoke Hants, UK) was selected, since it is a product exhibiting good clarity, controlled gelation and melting temperature, good diffusion characteristics, absence of toxic bacterial inhibitors, and relative absence of metabolically useful minerals and compounds. Different electrical and thermal properties of the agar must be accounted for in ohmic heating generated by PEF. While its density was assumed to be known $\rho=998.2\left(\mathrm{~kg} / \mathrm{mm}^{3}\right)$, its specific heat $c_{p}(\mathrm{~J} / \mathrm{kgK})$ and its thermal conductivity $k$ $(\mathrm{W} / \mathrm{mK})$ were determined experimentally in our lab; their functional dependence on the temperature (Eq.(3) and Eq.(2), respectively) was determined by linear regression (calibration not shown).

$$
\begin{array}{r}
k(T)=-7.317 \cdot 10^{-1}+7.322 \cdot 10^{-2} \cdot T-9.492 \cdot 10^{-6} \cdot T^{2} \\
c_{p}(T)=3.991 \cdot 10^{4}-423.184 \cdot T+1.879 \cdot T^{2}-3.716 \cdot 10^{-2} \cdot T^{3}+2.762 \cdot 10^{-6} \cdot T^{4}
\end{array}
$$

where $\mathrm{T}$ is the temperature of the agar.

In the case of the parameter of electrical conductivity, $\sigma(\mathrm{mS} / \mathrm{cm})$ Eq.(8), an experimental determination thereof was performed due to its dependency on temperature, which varies, in turn, with the application of PEF based on Eqs. (4) and (5): 


$$
\begin{aligned}
T_{f} & =T_{0}+\frac{W_{\text {total }}}{4.18} \\
W_{\text {total }} & =W_{j} \cdot N_{\text {pulses }}
\end{aligned}
$$

where $T_{f}$ and $T_{0}$ are the final and initial temperatures in ${ }^{\circ} \mathrm{C}, 4.18$ is a conversion factor from Joules to calories, $N_{\text {pulses }}$ is the number of squared pulses applied in the treatment, $W_{j}(\mathrm{~J} / \mathrm{kg})$ the specific energy per pulse that is calculated from Eq. (6), and $W_{\text {total }}$ is the total specific energy applied with the PEF treatment.

$$
W_{j}=\frac{V \cdot I \cdot t_{e f f}}{m}=\frac{V^{2} \cdot \tau \cdot n}{G A P \cdot \pi \cdot r^{2} \cdot \rho \cdot R}
$$

where in the left term $m$ is the mass of the product $(\mathrm{kg}), V$ is the applied voltage $(V), I$ is the current intensity generated by the electric field applied $(A)$, and $t_{\text {eff }}$ is the effective time in which the the electric field is actually applied $(s)$. In the right term, $\tau$ is the width of the pulse in which the electric field acts $(s), n$ is the number of pulses, GAP is the distance between electrodes (length of the chamber in $m$ ), $r$ is the radius of the electrodes $(\mathrm{m}), \rho$ is the density $\left(\mathrm{kg} / \mathrm{m}^{3}\right)$, and $R$ is the electrical resistance of the treatment chamber $(O h m s$, $\Omega)$ calculated as,

$$
R=\frac{G A P}{\pi \cdot r^{2} \cdot \sigma(T)}
$$

in which the experimentally determined electrical conductivity $\sigma$ finally appears.

From this analytical calculation, the relation between electrical conductivity, temperature, and the other electrical parameters becomes clear. Since for safe manipulation of the PEF device and to apply square wave pulses it is required to work with electric current within a range of 80 to $150 \mathrm{~A}$, it was necessary to define the relationship of the electrical conductivity of the agar with temperature in order to apply PEF within the working conditions of the PEF system. The measurement of the electrical conductivity of the agar consisted in preparing technical agars of different electrical conductivities by boiling distilled water with powder agar and different concentrations of $\mathrm{NaCl}$ added. Once dissolved, temperature and electrical conductivity were measured at different temperatures (ranging between 50 and $90{ }^{\circ} \mathrm{C}$ ) with a type-K thermocouple (Ahlborn 
Almemo, Munich, Germany) and a conductivity probe (FYA641LFP1, Ahlborn Almemo) with temperature compensation connected to a data-logger (2590A, Ahlborn Almemo). With the obtained data, the following quadratic polynomial (response surface), Eq. (8) was generated by multiple regression analysis to ascertain the effect of temperature $(T)$ and percentage of $\mathrm{NaCl}$ ([\%]salt) on electrical conductivity $\sigma(T)$ using the DesignExpert 6.0.6 software package (Stat-Ease Inc. Minneapolis, MN, USA):

$$
\begin{array}{r}
\sigma(T)=-3.269 \cdot 10^{-1}+1.044 \cdot 10^{-2} \cdot T+9.250 \cdot[\%]_{\text {salt }}+2.940 \cdot 10^{-1} \cdot T \cdot[\%]_{\text {salt }} \\
+2.42 \cdot[\%]_{\text {salt }}^{2}+1.330 \cdot 10^{-3} \cdot T^{2} \cdot[\%]_{\text {salt }}-3.951 \cdot 10^{-2} \cdot T \cdot[\%]_{\text {salt }}^{2}
\end{array}
$$

Based on this equation, for a PEF working current intensity of $80 \mathrm{~A}$, an initial temperature of $23{ }^{\circ} \mathrm{C}$ in the agar, and an approximated initial electrical conductivity of $0.373 \mathrm{~S} \mathrm{~m}^{-1}$, the required concentration of salt is $0.24 \%$. In this way, the electrical conductivity of the sample is controlled to avoid exceeding $150 \mathrm{~A}$ by the end of the PEF treatments. To evaluate the temperature in different locations of the agar cylinders, a technical agar cylinder of $10-\mathrm{cm}$ height and $10-\mathrm{cm}$ diameter with $0.24 \%$ added $\mathrm{NaCl}$ was prepared. From this large cylinder, and using a hole puncher of 2-cm of diameter, small agar cylinders were obtained and cut in pieces of 2-cm height, which were subsequently introduced in the treatment chamber. For each PEF treatment, an agar cylinder was introduced in the treatment chamber contacting both electrodes. Three 1-mm-diameter fiber optic probes model TPT-62-BA-C7-F2-M2-R1-ST (Fiberoptic Components, USA) were introduced through three holes of 2-mm diameter with the purpose of registering the temperature at different locations of the agar cylinder. Figure 2 shows the different locations at which probes were located: i.e., at the center of the cylinder (P2), at the center of the cylinder and 1-mm from the transition between the electrode and the sample (P1), or at 1-mm from the transition between the sample, the electrode, and the Teflon cylinder (P4). The evolution of temperature at the different locations was recorded to evaluate the amount of heating produced when applying pulses of different electric field strengths $(2.5$ to $3.75 \mathrm{kV} / \mathrm{cm}$ ) and number of pulses (10 to 3000 pulses) through time (pulses applied up to a maximum of 60 seconds) at a frequency of $50 \mathrm{~Hz}$. Heating rates (expressed as the increase in number of degrees Celsius per second of PEF treatment, ${ }^{\circ} \mathrm{C} / \mathrm{s}$ ) for each PEF treatment condition at each location were determined from the slope of the relationship between the temperatures of the PEF-treated 
agar cylinder and heating time.

[Figure 2 about here.]

\subsection{Microbial inactivation by thermal treatments}

The strain of Salmonella Typhimurium 878 used in this investigation was supplied by the Spanish Type Culture Collection. In the course of this investigation, the culture was maintained on slants of Tryptic Soy Agar (Biolife, Milan, Italy) with 0.6\% Yeast Extract added (Biolife) (TSAYE). A broth subculture was prepared by inoculating a test tube containing $5 \mathrm{~mL}$ of Triptic Soy Broth (Biolife) with $0.6 \%$ Yeast Extract (TSBYE) with a single colony, followed by incubation at $37^{\circ} \mathrm{C}$ for $24 \mathrm{~h}$. With this subculture, a flask containing $50 \mathrm{~mL}$ of sterile TSBYE was inoculated to a final concentration of approximately $10^{6}$ cells $/ \mathrm{mL}$. The culture was incubated under agitation at $37^{\circ} \mathrm{C}$ until the stationary growth phase was reached $(24 \mathrm{~h})$, achieving a concentration of $2 \cdot 10^{9}$ $\mathrm{CFU} / \mathrm{mL}$ (data not shown). This suspension was used to define the heat resistance of Salmonella Typhimurium at isothermal conditions in buffers, and when treated by PEF in agar cylinders.

\subsubsection{Microbial inactivation at isothermal conditions}

In order to estimate the microbial inactivation that could be achieved in the different monitored temperature positions of the agar cylinder when applying PEF, heat resistance of Salmonella Typhimurium 878 was determined at isothermal conditions. For this, heat treatments were carried out in a thermoresistometer TR-SC (Condon, Lopez, Oria, \& Sala, 1989, Condon, Arrizubieta, \& Sala, 1993) in which microorganisms were treated at constant temperatures ranging from 55 to $64^{\circ} \mathrm{C}$ in sterilized $\mathrm{pH} 6.8 \mathrm{McIlvaine}$ citrate-phosphate buffer. Once the treatment medium was tempered, $0.2-\mathrm{mL}$ of the microbial suspension was inoculated into the treatment medium. At different heating times, 0.1-mL samples were collected and immediately pour-plated. Survival curves (decimal logarithm of the number of surviving microorganisms vs. heating time) were obtained at different investigated temperatures. From the obtained survival curves, the traditional decimal reduction time value ( $D_{t}$ value), i.e., the time to inactivate $90 \%$ of the microbial population, and the $z$ value, i.e., the temperature increase to reduce the $D_{t}$ value by $90 \%$, were calculated.

Based on the heating rates obtained when PEF treatments were applied, temperatures at different points of the agar through time (non-isothermal treatments) were estimated. The estimated survival curves corresponding 
to non-isothermal treatments at the corresponding point of the agar cylinder were calculated by integrating the lethal effect of the different temperatures for each treatment time ( $\mathrm{L}$ value) and applying the following equation:

$$
L=\int_{0}^{t^{\prime}} \frac{t}{D_{\text {Tref }} \cdot 10^{\frac{T_{r e f}-T}{z}}} \cdot d t
$$

where $t^{\prime}$ is the PEF heating time (in seconds), and $D_{\text {Tref }}$ is the $D_{t}$ value at a reference temperature $\left(T_{r e f}\right)$ : in this investigation, $60^{\circ} \mathrm{C}$, obtained under isothermal conditions.

\subsubsection{Microbial inactivation in solid agar}

In order to validate the estimated microbial inactivation based on Eq. 9, the lethality of PEF treatments in agar cylinders was determined. For this purpose and prior to treatments, $1 \mathrm{~mL}$ of the microbial suspension was added to $1 \mathrm{~L}$ of sterilized technical agar with $0.24 \% \mathrm{NaCl}$ when the liquid agar was at $47^{\circ} \mathrm{C}$, which is not lethal for the pathogen but allows for a homogeneous distribution of the population over the entire sample. Subsequently, the agar was cooled down until gelification. With a sterile hole puncher of 2-cm of diameter, small agar cylinders were obtained and cut in pieces of 2-cm height, which were introduced in the treatment chamber. Two sets of experimental conditions designed to investigate the influence of temperature on microbial inactivation by PEF were applied: i) $2.5 \mathrm{kV} / \mathrm{cm}$ electrical field strength, 3000 squared pulses of $3 \mu s$, at a frequency of $50 \mathrm{~Hz}$ during 60 seconds; ii) $3.75 \mathrm{kV} / \mathrm{cm}$ electrical field strength, 3000 pulses of $3 \mu$ s at a frequency of $50 \mathrm{~Hz}$ during 60 seconds. After treatments, the agar cylinder was extracted from the treatment chamber in sterile conditions. Pieces of $0.2 \mathrm{~g}$ of agar from positions P2 and P4 were added to $1 \mathrm{~mL}$ of sterile $0.1 \%$ peptone water, homogenized, and $0.1 \mathrm{~mL}$ thereof were plated onto TSAYE. Plates were incubated at $37^{\circ} \mathrm{C}$ for $24 \mathrm{~h}$, after which colonies were counted with an improved image analyzer automatic counter (Protos, Analytical Measuring Systems, Cambridge, UK) as described elsewhere (Condon et al., 1996). Survival curves were based on mean values obtained from three independent experiments.

\subsection{Finite Element model for ohmic heating}

A coupled thermo-electrical problem is involved in ohmic heating. On the one hand, Laplace equations solve the intensity of the electric field applied over the domain. On the other hand, Joule's equation determines the internal energy generated by the electric field. According to Joule's equation, the heat generated $Q(J)$ 
during ohmic heating is proportional to the square of the electrical current that flows through the sample, its resistance, and the time in the course of which such current is flowing.

The governing equation for heat transfer is,

$$
\rho c_{p}(T) \frac{\partial T}{\partial t}-\nabla \cdot(k(T) \nabla T)=Q
$$

where $\rho$ is the density of the solid, $c_{p}(T)$ is the specific heat, and $k(T)$ is the thermal conductivity. The term, $Q$ is the the conversion of electrical to thermal energy (Joule heating),

$$
Q=\sigma(T) \mathbf{E}^{2}
$$

where $\mathbf{E}$ denotes the electric field strength. It is assumed that the pulsating electric field does not induce a time varying magnetic field, thus $\nabla \times \mathbf{E}=0$. As a consequence, the electric field vector $\mathbf{E}$ can be written as the gradient of the electrical potential $V$,

$$
\mathbf{E}: \mathbf{E}-\nabla V=0
$$

Based on charge conservation, the governing equation for the electrical potential can be written as

$$
\nabla \cdot \mathbf{J}=\nabla \mathbf{J} \cdot[\sigma(T) \nabla V]=0
$$

with $\mathbf{J}$ denoting the current density.

The coupled differential problem can be solved by the finite element method. To simulate ohmic heating, we developed an axisymmetric model using the commercial software Abaqus (Dassault Systèmmes). The model includes three different parts: the electrodes of variable cross-section (radius of 10-mm and thickness of 2$\mathrm{mm}$ ), the plastic container that houses the agar and the electrodes, and the treatment chamber containing the technical agar with 20-mm diameter and 20-mm length (Figure 3.a). The number of nodes and elements for discretization of the domain were 3,321 and 1,050, respectively. The model was meshed with 8-node quadrilateral finite elements with quadratic approximation (DCAX8E). 
Material properties for the agar were the ones described previously, presenting dependence on temperature for electrical $(\sigma)$ and thermal $(k)$ conductivity and specific heat $\left(c_{p}\right)$. Material properties for electrodes were those of stainless steel and for the plastic envelope those of teflon (Table 1).

[Table 1 about here.]

Two different types of boundary conditions were introduced in the model: electrical and thermal.

First, the electric potential between electrodes was defined by setting one electrode as the voltage electrode (in blue in Figure 3.b) and the other as the ground electrode (in green in Figure 3-b). The load amplitude of the electric pulse was the same as the one used in the commercial PEF device, i.e., a squared electrical pulse of $3 \mu \mathrm{s}$ of duration, a given number of pulses, frequency, and total duration of the experiment. Knowing this, each calculation step has a plateau of $3 \mu \mathrm{s}$ in which the electric pulse is set to the highest voltage (i.e., $5 \mathrm{kV}$ - $2.5 \mathrm{kV} / \mathrm{cm}$ - or $7 \mathrm{kV}-3.75 \mathrm{kV} / \mathrm{cm}$ - depending on the experiment under analysis), followed by a valley of variable duration (i.e., depending on the number of pulses, frequency, and duration of the experiment) in which no electric field is applied.

Secondly, a condition of natural convection was set in the boundary of the domain in order to allow the heat to be transferred to the surrounding media and to allow the sample to cool down. Natural convection boundary conditions depend on the difference between the temperature in the surrounding media and the domain, and a coefficient of heat transfer $h(W / m K)$. For air, values for $h$ in natural convection are relatively low; in this study, they were set to $h=5 \mathrm{~W} / \mathrm{mK}$. Finally, the initial temperature was $T_{0}^{\text {Agar }}=23^{\circ} \mathrm{C}$ for the technical agar, $T_{0}=25{ }^{\circ} \mathrm{C}$ for the electrodes, and $T_{0}=25{ }^{\circ} \mathrm{C}$ for the plastic container. Simulated temperatures were recorded at the points at which the real probe should be located in the experiments: i) at the center of the treatment chamber (P2); and ii) at 1-mm from the transition between the sample, electrode, and plastic cylinder (P4).

For the sake of simplicity and computational effort, we assume that the treatment chamber presents a perfect symmetry of revolution (i.e., the use of an axisymmetric model); the control unit is able to deliver perfect continuum pulses during all the treatment; the material behavior is perfectly homogeneous and there is not air entrapped, nor gaps between the electrodes and the agar; and that the probes do not affect the conductivity 
and/or electric field during measurement. Despite these assumptions, there is not lack of generality in the present benchmark study.

\section{Results and Discussion}

This study was the first to evaluate microbial inactivation via PEF treatments in a solid product based on generated ohmic heating, considering Salmonella Typhimurium as reference. Moreover, inactivation in a solid (an agar cylinder) has been estimated based on the isothermal heat resistance of Salmonella Typhimurium after the application of PEF treatments and the time-temperature distribution predictions obtained by numerical simulation tools, after which the results of the actual microbial inactivation were validated. As indicated, it was necessary to define the ohmic heating rates in the agar cylinder used as a reference solid material when applying PEF under different conditions in order to define the hottest and coldest point to which the results refer. These rates were estimated via computational simulation by predicting temporal space distribution of the temperature, a procedure that is essential in order to predict distribution in a static ohmic heating chamber, for example, and to optimize ohmic heating processes (Knoerzer, Regier, \& Schubert, 2006).

\subsection{Experimental results for ohmic heating and microbial inactivation}

In a first step, temperature increment in the different tested points of the agar cylinder when applying $\mathrm{PEF}$ treatments of $2.5 \mathrm{kV} / \mathrm{cm}$ at $50 \mathrm{~Hz}$ was evaluated (Figure 4). Linear relationship between processing time (number of pulses multiplied by pulse frequency) and temperature increase were described. As observed, points $\mathrm{P} 2, \mathrm{P} 5$ and $\mathrm{P} 6$ were the ones with the highest heating rates $\left(1.14 \pm 0.04,1.28 \pm 0.08,1.16 \pm 0.06{ }^{\circ} \mathrm{C} / \mathrm{s}\right)$, while points $\mathrm{P} 1$ and $\mathrm{P} 4$ were the ones with the lowest $\left(0.99 \pm 0.02,0.95 \pm 0.03{ }^{\circ} \mathrm{C} / \mathrm{s}\right)$. There were no statistically significant differences $(\mathrm{p}=0.05)$ among slopes, even comparing positions ( $\mathrm{P} 4$ and $\mathrm{P} 5)$ with the most distinct values, but differences of almost $10{ }^{\circ} \mathrm{C}$ were determined after 20 seconds of treatment among the hottest and the coldest points. This means that the longer the treatment time, the lesser the uniformity of temperature in the cylinder. Considering this last appreciation and in order to evaluate the effect of the different heating rates in terms of lethality, Salmonella Typhimurium inactivation was evaluated in position P4, the coldest point, after applying PEF treatments of different duration. Also, position P2 (the central point of the agar cylinder), which presented one of the highest heating rates, was selected to compare results and to evaluate the 
uniformity of heating. It has to be pointed out that position $\mathrm{P} 4$, and also position P1, i.e., the ones with the lowest heating rates, were located close to the high voltage electrode. Similar observations have been reported in literature indicating a cold zone at the junction of electrodes in ohmic heating treatments (Marra, 2014). The occurrence of these cold zones is a noticeable limitation of PEF technology in terms of its application to design pasteurization or even sterilization treatments.

[Figure 4 about here.]

In order to evaluate the lethality of PEF in positions $\mathrm{P} 2$ and $\mathrm{P} 4$, the heating rates shown in Figure 4 were used to estimate temperature fluctuation over time when applying PEF at $2.5 \mathrm{kV} / \mathrm{cm}$ and $50 \mathrm{~Hz}$ (Figures 5.a and 5.b for positions $\mathrm{P} 2$ and $\mathrm{P} 4$, respectively). Also, and since ohmic heating is proportional to the square of electric field strength (Eq. 1), temperature increase was evaluated at a higher field strength of $3.75 \mathrm{kV} / \mathrm{cm}$ and $50 \mathrm{~Hz}$ (Figures 5.c and 5.d for positions P2 and P4, respectively). In this case, heating rates were $2.52 \pm 0.34$ and $2.15 \pm 0.22{ }^{\circ} \mathrm{C} / \mathrm{s}$ for positions $\mathrm{P} 2$ and $\mathrm{P} 4$ respectively.

Based on these time-temperature profiles and on a $D_{60^{\circ} \mathrm{C}}$ value of $0.39 \pm 0.04^{\prime}$ and a $z$ value of $5.0 \pm 0.1$ ${ }^{\circ} \mathrm{C}$ obtained from the thermal inactivation of Salmonella Typhimurium at isothermal conditions in McIlvaine buffer of pH 6.8 (data not shown), Figure 5 was plotted for P2 (Figure 5.a and 5.c) and P4 (Figure 5.b and 5.d) for treatments at 2.5 and $3.75 \mathrm{kV} / \mathrm{cm}$. In this figure, the average (dotted lines), and the maximum and minimum theoretical temperatures (pink zone) when applying PEF were calculated based on heating rates. The theoretical inactivation of Salmonella Typhimurium (continuous line) was calculated using (9). In this graph, the rate of inactivation for the maximum and minimum temperatures after a given PEF treatment (blue zone) was also calculated. As observed, inactivation speed was faster the higher the temperature, and it was more rapid in position $\mathrm{P} 2$ than in $\mathrm{P} 4$ at both field strengths.

In the case of $2.5 \mathrm{kV} / \mathrm{cm}$, after 48 seconds of PEF treatments 5 - $\log _{10}$ cycles of Salmonella Typhimurium were inactivated in the center of the cylinder (Figure 5.a), whereas in the best-case scenario only two cycles were inactivated at a distance of 1-mm from the high voltage electrode (Figure 5.b), thereby registering mean temperatures of $73{ }^{\circ} \mathrm{C}$ (maximum $78{ }^{\circ} \mathrm{C}$ ) and $63{ }^{\circ} \mathrm{C}$ (maximum $73{ }^{\circ} \mathrm{C}$ ), in positions $\mathrm{P} 2$ and $\mathrm{P} 4$, respectively. To achieve 5 - $\log _{10}$ cycles of inactivation near the electrode, heating time had to be increased to 58 seconds by applying 2900 pulses of $3 \mu \mathrm{s}$, reaching mean temperatures of $75{ }^{\circ} \mathrm{C}$ in the worst-case scenario. In Figure 
5, the uniformity of the PEF treatments depending on location can likewise be easily observed. Thus, for an average $5-\log _{10}$ reduction of the microbial population, 44 and $50 \mathrm{~s}$ would be required in positions $\mathrm{P} 2$ and $\mathrm{P} 4$, respectively, thereby determining possible variations in temperature in those locations from 64 to $73^{\circ} \mathrm{C}\left(9^{\circ} \mathrm{C}\right)$ in position $\mathrm{P} 2$ and from 62 to $75^{\circ} \mathrm{C}\left(13^{\circ} \mathrm{C}\right)$ in position $\mathrm{P} 4$. From a practical point of view, such a variation in temperature and heating rates would imply a variation in processing time of 8 seconds (from 40 to $48 \mathrm{~s}$ ) and 14 seconds (from 44 to $58 \mathrm{~s}$ ) in positions P2 and P4, respectively, in order to achieve 5-Log 10 -reductions of Salmonella Typhimurium.

When evaluating the effect of electric field strength, conclusions similar to those reached at $2.5 \mathrm{kV} / \mathrm{cm}$ can be obtained at $3.75 \mathrm{kV} / \mathrm{cm}$, but with more rapid temperature increment and microbial inactivation. Thus, 5- $\log _{10}$ reductions were obtained after 29 seconds of treatment (a $50 \%$ reduction of processing time for a $1 \mathrm{kV} / \mathrm{cm}$ increase in field strength) independently of location, with less variation in time for this level of inactivation, requiring from 18 to 23 seconds $(5 \mathrm{~s})$ and from 20 to 29 seconds $(9 \mathrm{~s})$ in positions $\mathrm{P} 2$ and P4, respectively. These results seem to indicate a higher uniformity in temperature distribution when electric field strength is increased. However, when measuring temperature experimentally, the variation of this parameter seems to be larger than when applying $2.5 \mathrm{kV} / \mathrm{cm}$. Thus for an average 5 - $\log _{10}$ microbial reduction at $3.75 \mathrm{kV} / \mathrm{cm}$, temperature varied from 63 to $75{ }^{\circ} \mathrm{C}\left(12{ }^{\circ} \mathrm{C}\right.$ after $\left.19.5 \mathrm{~s}\right)$ in position $\mathrm{P} 2$ and from 60 to $79{ }^{\circ} \mathrm{C}\left(19{ }^{\circ} \mathrm{C}\right.$ after $\left.23 \mathrm{~s}\right)$ in position $\mathrm{P} 4$, respectively, which was higher than when $\mathrm{PEF}$ was applied at $2.5 \mathrm{kV} / \mathrm{cm}$. These results would indicate that the faster the heating rate, the higher the uncertainty when measuring temperature experimentally, whereby the variability of measurements increases depending on small variations in the measurement point. This uncertainty is reflected in a wider range of temperatures (red zone in Figure 5) due to a higher variability in heating rates calculated from the experimental measurements $(95 \%$ confidence limits of the heating rates at $3.75 \mathrm{kV} / \mathrm{cm}$ were larger - e.g. 0.34 and 0.22 - than for $2.5 \mathrm{kV} / \mathrm{cm}$ - from 0.02 to 0.08 ).

In order to evaluate in more detail this possible influence of uncertainty regarding the location of the probe when measuring the temperature, numerical simulation tools can be of great interest, as will be discussed later on. In order to validate these results, Figure 5 also includes the actual inactivation of Salmonella Typhimurium obtained in positions P2 and P4 after different PEF treatments at both field strengths (marked with stars in Figure 5). As observed, the estimated degree of microbial inactivation agrees with the $\log _{10}$ reductions observed 
at both locations and field strengths: the most intense PEF treatments achieve 5- $\log _{10}$ reductions, and even situations without any detectable microbial growth (reductions of an order of more than 6 - $\log _{10}$ ). Inactivation was only slightly underestimated for the treatments at $3.75 \mathrm{kV} / \mathrm{cm}$ and position P2, which was the one with the highest variability in heating rates. The agreement between estimated and real inactivation would indicate that heat resistance obtained under isothermal conditions can adequately predict the inactivation of Salmonella Typhimurium under non-isothermal conditions, which occur when PEF is applied. This agreement could be explained by the supposition that high heating rates would limit the possible occurrence of thermal adaptation of Salmonella, as has been observed for other microorganisms such as Listeria monocytogenes at lower heating rates under standard heat treatments (Monfort et al., 2012).

On the other hand, this concordance between estimated and observed microbial inactivation would also indicate that PEF treatments applied at the studied field strengths would not produce any additional inactivation than that generated by a heat treatment. In other words, the applied field strength would not be sufficient to produce irreversible electroporation, or to sensitize Salmonella Typhimurium to the applied temperatures, as has been observed at higher field strengths (Saldaña et al., 2010). These results are not in agreement with those obtained by other authors, who achieved an additional inactivation by electroporation even at low field strengths (Machado, Pereira, Martins, Teixeira, \& Vicente, 2010, Park \& Kang, 2013). This could be associated with a possible reversible electroporation which could become irreversible by means of heating, thereby increasing the treatment's overall lethality (Kim, Choi, \& Kang, 2017). That difference in observed behavior could be ascribed to the fact that those results were obtained in liquid media, in which ohmic heating might generate undesired electrochemical species that lead to inactivation, as pointed out (Timmermans et al., 2019). Electrochemical reactions take place at the interface between the electrode and the liquid. In this investigation, that effect could be taking place, but since a solid medium was used, the potentially generated electrochemical species would remain in the interface, not affecting the entire treated product as would occur in a liquid medium.

[Figure 5 about here.]

\subsection{Temperature and inactivation predictions of the mathematical model}

The obtained results show the potential of PEF for microbial inactivation in solid products. However, as the evaluation of the effect of PEF parameters on temporal and spatial distribution of electric field strength and 
temperature, and thus likewise of the degree of microbial inactivation, are all difficult to measure. Numerical simulation provides us with an adequate tool to obtain this information, permitting a confirmation of the experimental results and, in this case the detection of the coldest point in the treatment zone. Figure 6 shows the finite element simulation of ohmic heating resulting when a PEF treatment $(2.5 \mathrm{kV} / \mathrm{cm}$ at $50 \mathrm{~Hz})$ is applied in the treatment chamber, presenting results of temperature gradient (Figure 6.a), heat transfer (Figure 6.b), and electric potential (Figure 6.c). As observed, numerical simulations support the hypothesis that, although electric potential is homogeneous and linear in the sample (Figure 6.c), the distribution of temperature presents a gradient of up to $10{ }^{\circ} \mathrm{C}$ towards the periphery (Figure 6.a), thereby representing a risk factor to account for in the context of PEF treatments. This could be probably be ascribed to heat transfer (Figure 6.b), which is mostly focalized in the electrodes due to the high thermal conductivity of stainless steel, whereby the chamber is almost adiabatic due to its plastic components.

[Figure 6 about here.]

Nevertheless, predictions of numerical simulations were in good agreement with experimental temperature ranges and inactivation values. Thus, Figure 7 plots simulated temperature increments and microbial inactivation in positions P2 (Figure 7.a and 7.c) and P4 (Figures 7.b and 7.d) after the application of PEF at 2.5 $\mathrm{kV} / \mathrm{cm}$ and $50 \mathrm{~Hz}$ (Figures 7.a and 7.b), and at $3.75 \mathrm{kV} / \mathrm{cm}$ and $50 \mathrm{~Hz}$ (Figures 7.c and 7.d). Lines indicate the predictions, and shaded areas represent the experimental ranges previously shown in Figures 5. As observed, simulated temperatures in the technical agar (black and blue solid lines in Figure 7) fell inside the experimental ranges for the center (position 2) and the 'corner' of the treatment chamber (position 4). Moreover, predicted inactivation based on simulated temperatures fell inside the experimental range (grey shaded areas in Figure $7)$.

Interestingly, models help to outline the measuring system's high sensitivity to the location of the probe, which could help explain the high variability in some of the experimental measurements. Simulated temperatures (black and blue solid lines in Figure 7) correspond to the same spatial location within the treatment chamber (positions P2 and P4), with a slight perturbation of $0.5-\mathrm{mm}$ around the measuring point. This small perturbation, which simulates the uncertainty in the positioning of the temperature probe, shows that it is extremely important to ensure a precise measuring protocol in order to properly determine the gradient in the 
temperature field within the sample. This is more important the higher the heating rate, in our case when a more intense PEF treatment was applied $(3.75 \mathrm{kV} / \mathrm{cm})$. In the simulations, it seemed that a lesser degree of variability was to be estimated when a higher field strength was applied at both locations. That variability can be observed in more detail in Figure 8, which features the correlation between the experimental final temperatures in the chamber ( $\mathrm{P} 2$ and $\mathrm{P} 4)$ and the simulated final temperature after the application of PEF treatments at 2.5 and $3.75 \mathrm{kV} / \mathrm{cm}$.

As observed, although occasionally the experimental temperature is slightly overestimated, simulated temperatures lie within a suitable range and, thus, models can be used to explore different treatments without having to recur to experimental benchwork. Vertical error-bars in Figure 8 show the degree of uncertainty in experimental measurements, and horizontal error-bars indicate the uncertainty in numerical measurements (for experiments of different treatment times). As observed, numerical models showed that the uncertainty in temperature due to the uncertainty in the positioning of the temperature probe was higher in P4 (the zone close to the electrode) than in P2 (center) and, again, that a higher electric potential helped to reduce the gradient of temperature thanks to shorter application times. That is, the application of pulses of higher electric field strength would result in a more homogeneous temperature distribution by limiting the release of heating through electrodes.

[Figure 7 about here.]

[Figure 8 about here.]

\section{Conclusions}

This study demonstrated the potential of PEF as a system capable of rapidly achieving microbial inactivation in solid products thanks to a higher heat capacity transfer when applying field strengths over $1 \mathrm{kV} / \mathrm{cm}$. Based on our knowledge, this is the first study on this particular aspect, and it was possible to reduce 5 or even more $\log _{10}$ cycles of Salmonella Typhimurium 878 in solid agar with treatment times below 1 minute. These results indicated that PEF could be further investigated and considered as an alternative to traditional conductive heat treatments for microbial inactivation. However, gradients of up to $10^{\circ} \mathrm{C}$ were determined between the hottest and coldest point which is located in the interphase zone between agar and the high voltage electrode. 
The consequence is a need for experimental times with delays of around 10 seconds to ensure a certain level of microbial inactivation. Therefore, it is essential to evaluate the effect of PEF parameters on heating rates and temperature uniformity. For that evaluation, numerical models proved to be a useful tool. Based on the obtained simulations, increasing electric field strength reduced processing time (an increment of $1 \mathrm{kV} / \mathrm{cm}$ resulted in half processing time, i.e., 58 to 29 seconds), as well as the temperature distribution in the agar cylinder, thereby improving treatment uniformity and, consequently, the degree of microbial inactivation. However, more research is necessary in order to evaluate the effect not only of electric field strength but of pulse width, frequency, and other factors on temperature and microbial inactivation uniformity in the entire solid.

\section{Acknowledgements}

The authors thank A. Picardo for his help in designing and constructing the PEF treatment chamber.

The authors gratefully acknowledge research support from the Spanish Ministry of Economy and Competitiveness (Grant GL2017-84084-R and DPI2017-84047-R ) and the Department of Industry and Innovation (Government of Aragón) through Research Group Grant T24-17R and A03-17R (cofinanciado con Feder 20142020: Construyendo Europa desde Aragón).

The authors also acknowledge assistance provided by CIBER-BBN, an initiative funded by the VI National R \& D \& i Plan 2008-2011, Iniciativa Ingenio 2010, Consolider Program, CIBER Actions, and financed by the Instituto de Salud Carlos III with assistance from the European Regional Development Fund.

\section{References}

Barbosa-Canovas, G. V., Fernandez-Molina, J. J., Swanson, B. G., 2001. Pulsed electric fields: A novel technology for food preservation. Agro Food Industry Hi-Tech 12 (2), 9-14.

Buckow, R., Ng, S., Toepfl, S., September 2013. Pulsed electric field processing of orange juice: A review on microbial, enzymatic, nutritional, and sensory quality and stability. Comprehensive Reviews in Food Science and Food Safety 12 (5), 455-467. 
Condon, S., Arrizubieta, M. J., Sala, F. J., 1993. Microbial heat-resistance determinations by the multipoint system with the thermoresistometer TR-SC - improvement of this methodology. Journal of Microbiological Methods 18 (4), 357-366.

Condon, S., Lopez, P., Oria, R., Sala, F. J., 1989. Thermal death determination - design and evaluation of a thermoresistometer. Journal of Food Science 54 (2), 451-457.

Condon, S., Palop, A., Raso, J., Sala, F. J., 1996. Influence of the incubation temperature after heat treatment upon the estimated heat resistance values of spores of Bacillus subtilis. Letters in Applied Microbiology 22 (2), 149-152.

Gerlach, D., Alleborn, N., Baars, A., Delgado, A., Moritz, J., Knorr, D., 2008. Numerical simulations of pulsed electric fields for food preservation: A review. Innovative Food Science and Emerging Technologies 9 (4), $408-417$.

Kaur, N., Singh, A. K., 2016. Ohmic heating: Concept and applications-a review. Critical Reviews in Food Science and Nutrition 56 (14), 2338-2351.

Kim, S.-S., Choi, W., Kang, D.-H., 2017. Application of low frequency pulsed ohmic heating for inactivation of foodborne pathogens and ms-2 phage in buffered peptone water and tomato juice. Food Microbiology 63 , $22-27$.

Knoerzer, K., Regier, M., Schubert, H., 2006. Microwave heating: A new approach of simulation and validation. Chemical Engineering and Technology 29 (7), 796-801.

Machado, L. F., Pereira, R. N., Martins, R. C., Teixeira, J. A., Vicente, A. A., 2010. Moderate electric fields can inactivate Escherichia coli at room temperature. Journal of Food Engineering 96 (4), 520-527.

Marra, F., 2014. Mathematical model of solid food pasteurization by ohmic heating: Influence of process parameters. Scientific World Journal 2014 (236437).

McLaren, C. T., Kopatz, C., Smith, N. J., Jain, H., 2019. Development of highly inhomogeneous temperature profile within electrically heated alkali silicate glasses. Scientific Reports 9 (2805). 
Monfort, S., Sagarzazu, N., Gayan, E., Raso, J., Alvarez, I., 2012. Heat resistance of Listeria species to liquid whole egg ultrapasteurization treatment. Journal of Food Engineering 111 (2), 478-481.

Park, I.-K., Kang, D.-H., 2013. Effect of electropermeabilization by ohmic heating for inactivation of Escherichia coli O157:H7, Salmonella enterica Serovar Typhimurium, and Listeria monocytogenes in buffered peptone water and apple juice. Applied and Environmental Microbiology 79 (23), 7122-7129.

Saldaña, G., Puertolas, E., Condon, S., Alvarez, I., Raso, J., 2010. Modeling inactivation kinetics and occurrence of sublethal injury of a pulsed electric field-resistant strain of Escherichia coli and Salmonella Typhimurium in media of different ph. Innovative Food Science and Emerging Technologies 11 (2), 290-298.

Samaranayake, C. P., Sastry, S. K., Zhang, H., 2005. Pulsed ohmic heating - a novel technique for minimization of electrochemical reactions during processing. Journal of Food Science 70 (8), E460-E465.

Sastry, S., 2008. Ohmic heating and moderate electric field processing. Food Science Technology International $14(5), 419-422$.

Sastry, S. K., 2004. Advances in ohmic heating and moderate electric field (MEF) processing. CRC Press.

Sastry, S. K., Li, Q., 1996. Modeling the ohmic heating of foods. Food Technology 50 (5), 246-248.

Shim, J., Lee, S. H., Jun, S., 2010. Modeling of ohmic heating patterns of multiphase food products using computational fluid dynamics codes. Journal of Food Engineering 99 (2), 136-141.

Timmermans, R. A. H., Mastwijka, H. C., Berendsen, L. B. J. M., Nederhoff, A. L., Matser, A. M., Van Boekel, M. A. J. S., Groot, M. N. N., 2019. Moderate intensity pulsed electric fields (PEF) as alternative mild preservation technology for fruit juice. International Journal of Food Microbiology 298, 63-73.

Wölken, T., Sailer, J., Maldonado-Parra, F. D., Horneber, T., Rauh, C., 2017. Application of numerical simulation techniques for modeling pulsed electric field processing. Handbook of electroporation, 1-31.

Yildiz-Turp, G., Sengun, I. Y., Kendirci, P., Icier, F., 2013. Effect of ohmic treatment on quality characteristic of meat: A review. Meat Science 93 (3), 441-448. 


\begin{tabular}{|l|c|c|}
\cline { 2 - 3 } \multicolumn{1}{c|}{} & Stainless steel & Teflon \\
\hline$\sigma(\mathrm{W} / \mathrm{mK})$ & 12 & 0.25 \\
\hline$\sigma(S / m)$ & $1.45 \cdot 10^{6}$ & $10^{-24}$ \\
\hline$c_{p}\left(\mathrm{~J} \cdot \mathrm{kg}^{-1} \mathrm{~K}^{-1}\right)$ & 502 & 2,200 \\
\hline$\rho\left(\mathrm{kg} / \mathrm{m}^{3}\right)$ & 7,850 & 970 \\
\hline
\end{tabular}

Table 1: Properties for stainless steel and teflon. 


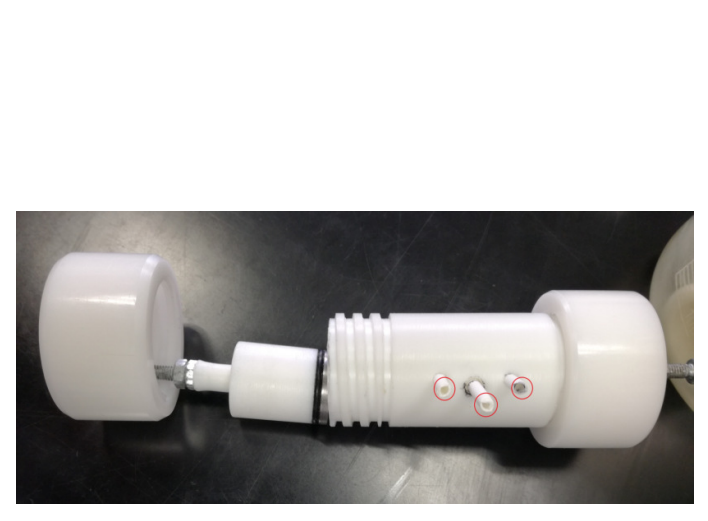

(a)

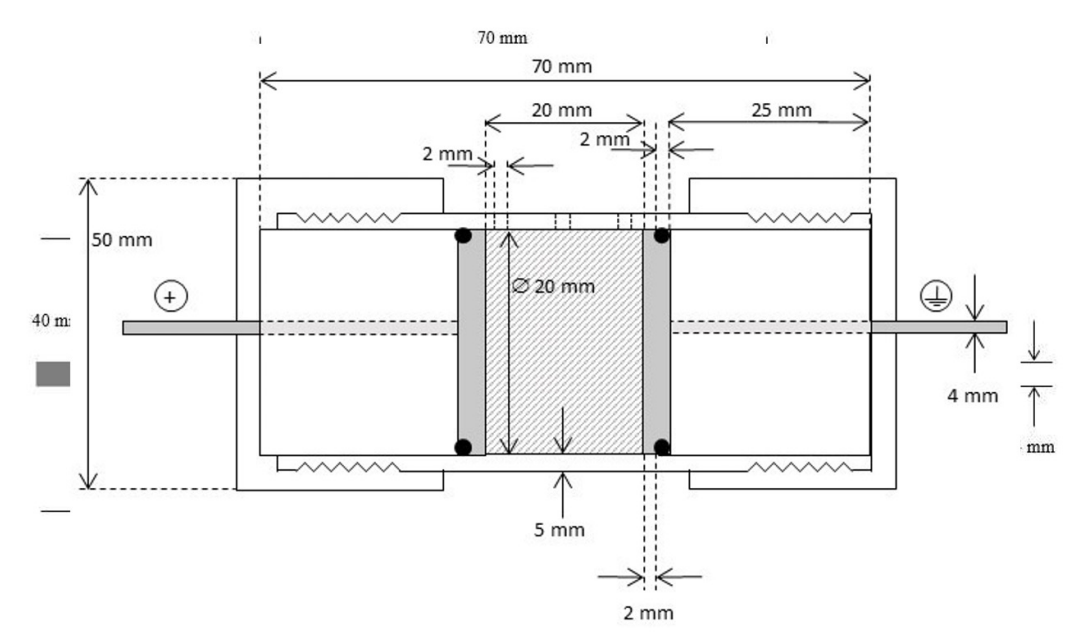

(b)

Figure 1: Treatment chamber used in the experimental set-up. (a) Photograph. (b) Scheme and dimensions. 


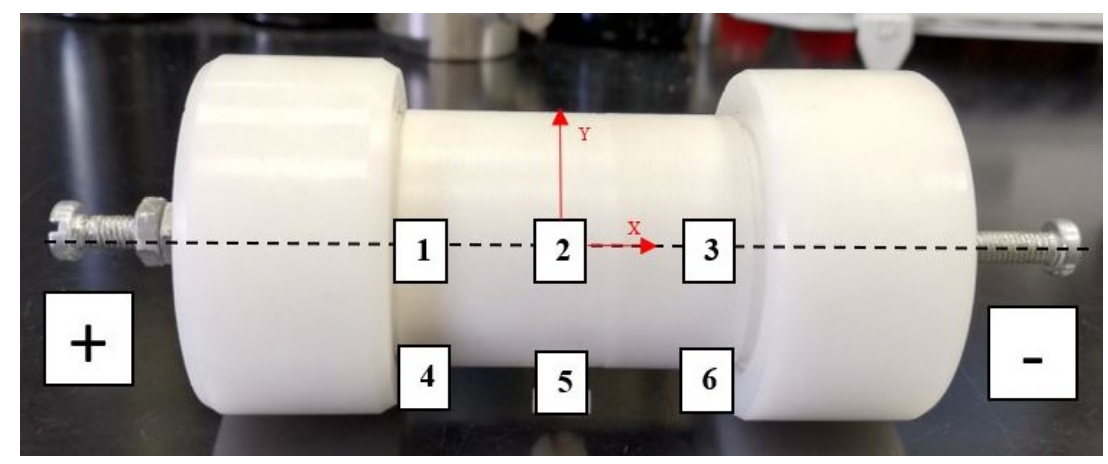

Figure 2: Location of the different temperature-measurement points in the PEF treatment chamber. 


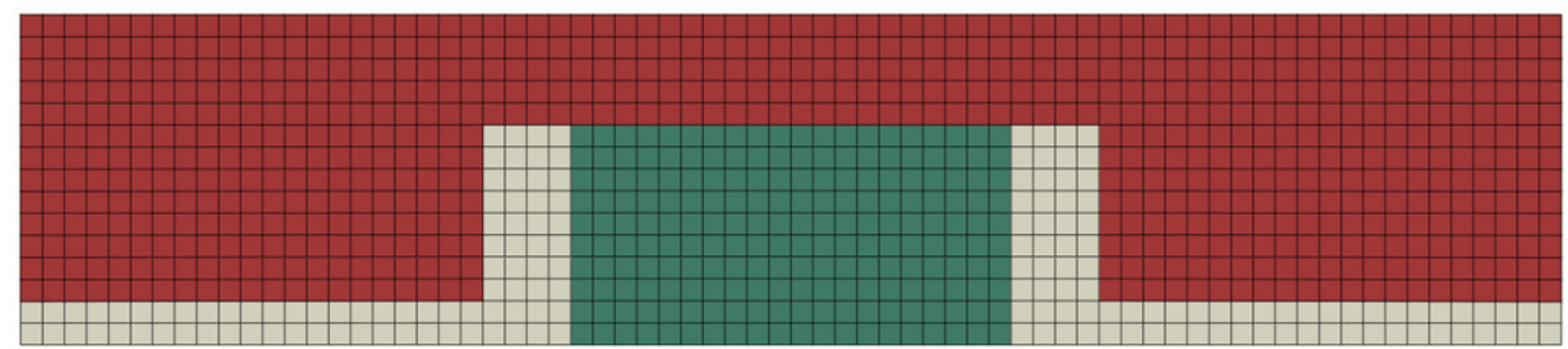

(a)

HEAT TRANSFER TO AIR

(NATURAL CONVECTION)

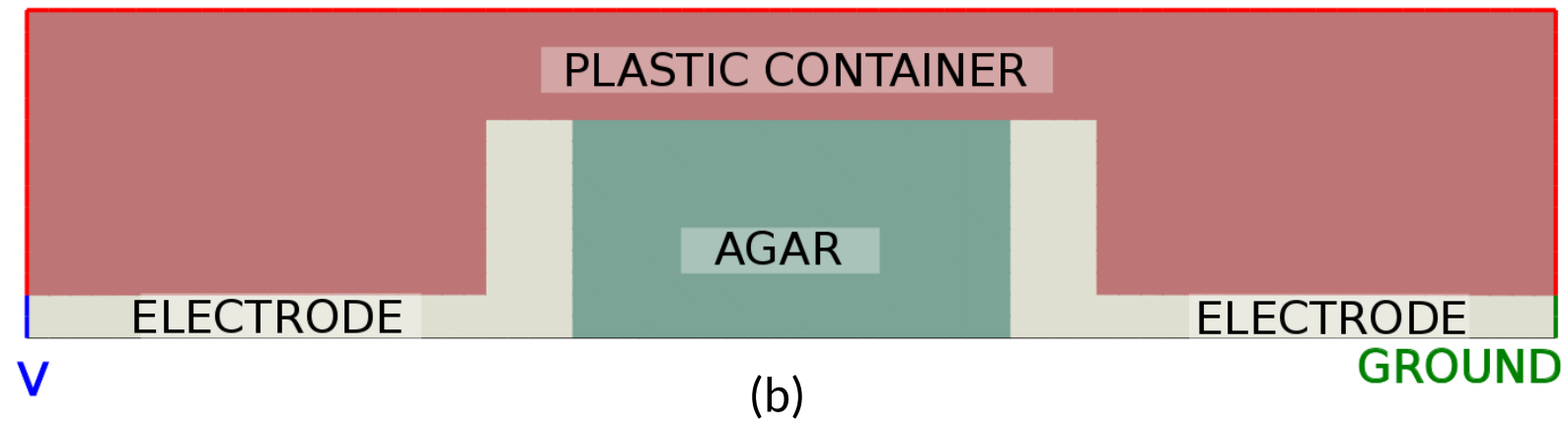

Figure 3: A finite element model of ohmic treatment. (a) Discretization of the domain including electrodes, plastic envelope, and agar; (b) Boundary conditions for the resolution of the coupled thermo-electrical problem. 


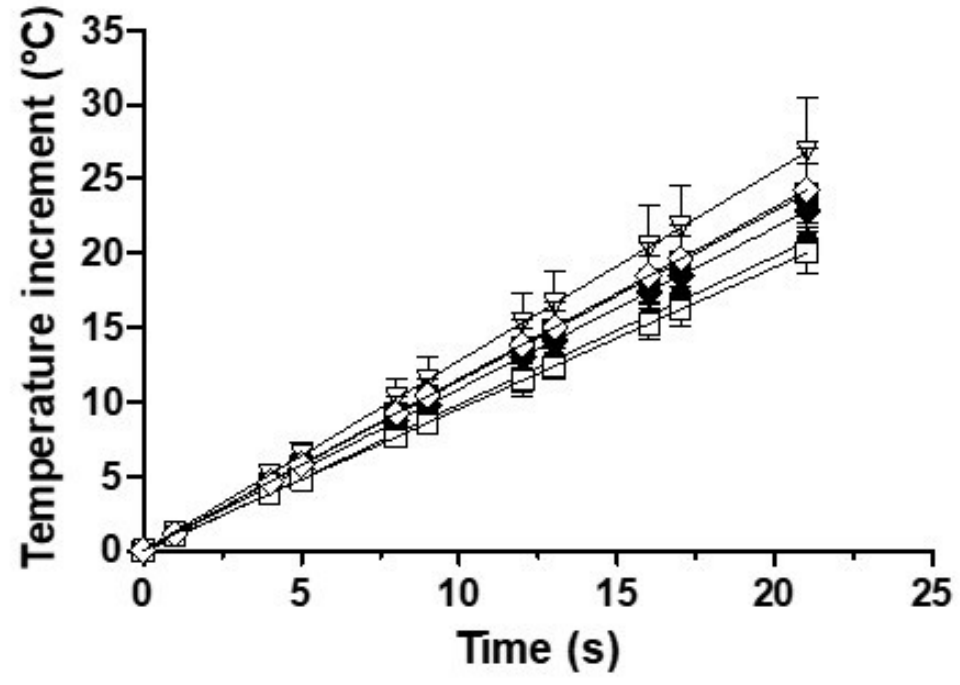

Figure 4: Increase in the temperature of the agar when applying PEF treatments of $2.5 \mathrm{kV} / \mathrm{cm}$ at $50 \mathrm{~Hz}$ at different positions of the agar: P1 $(\bullet)$, P2 $(\mathbf{\square})$, P3 $(\boldsymbol{\Delta})$, P4 (०), P5 $(\square)$, and P6 $(\triangle)$. 

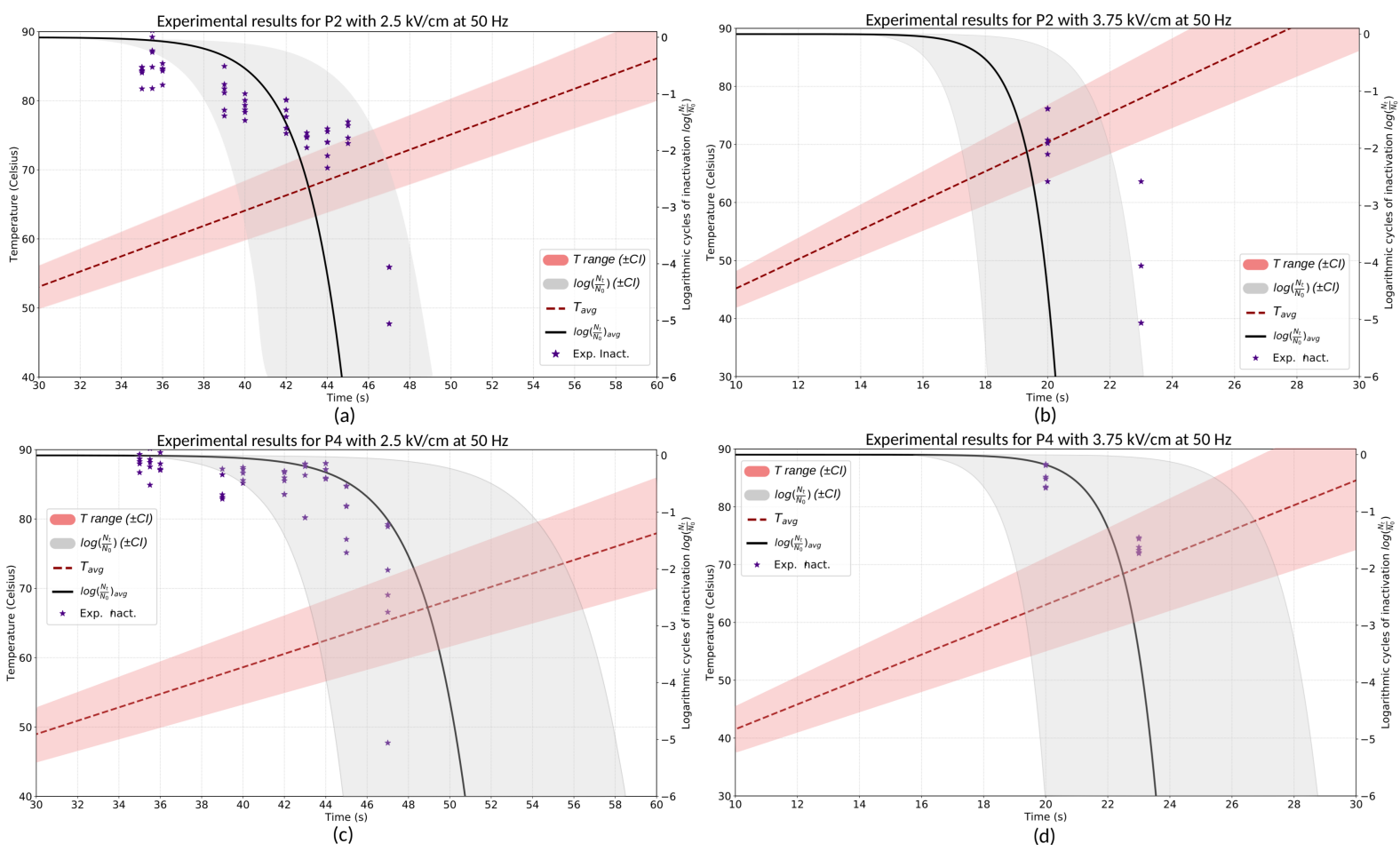

Figure 5: Results of experimental inactivation (inactivation showed in purple stars) and theoretical evolution of temperature (reddish palette) and inactivation (grayscale palette) of Salmonella Typhimurium in cylinders of agar when applying PEF treatments of 2.5 $\left(5 \mathrm{~A}\right.$ and $5 \mathrm{~B}$ ) and $3.75 \mathrm{kV} / \mathrm{cm}\left(\mathrm{c}\right.$ and d) at $50 \mathrm{~Hz}$ in positions $\mathrm{P} 2$ (a and c) and 4 (b and d). $\log _{10}$ cycles of actual inactivation $(\bullet)$ of Salmonella Typhimurium. 


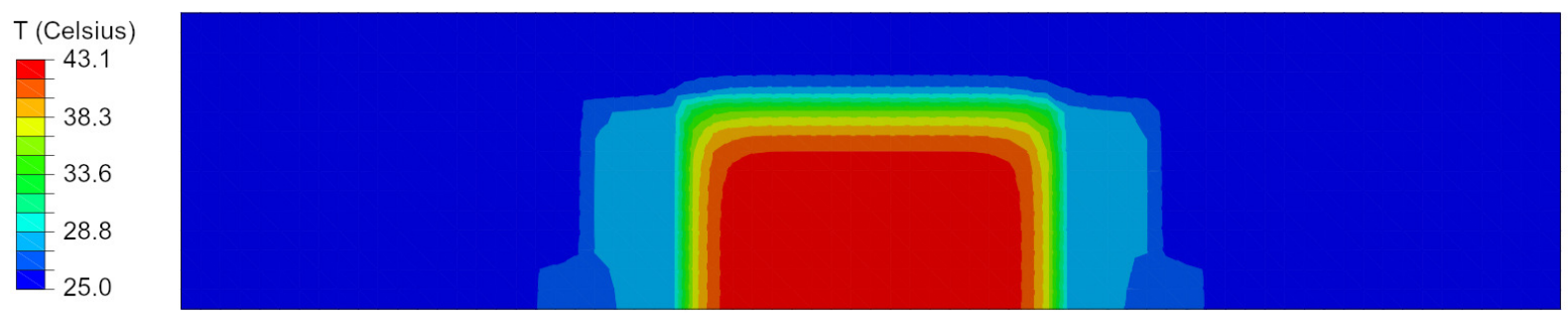

Electric

(a)
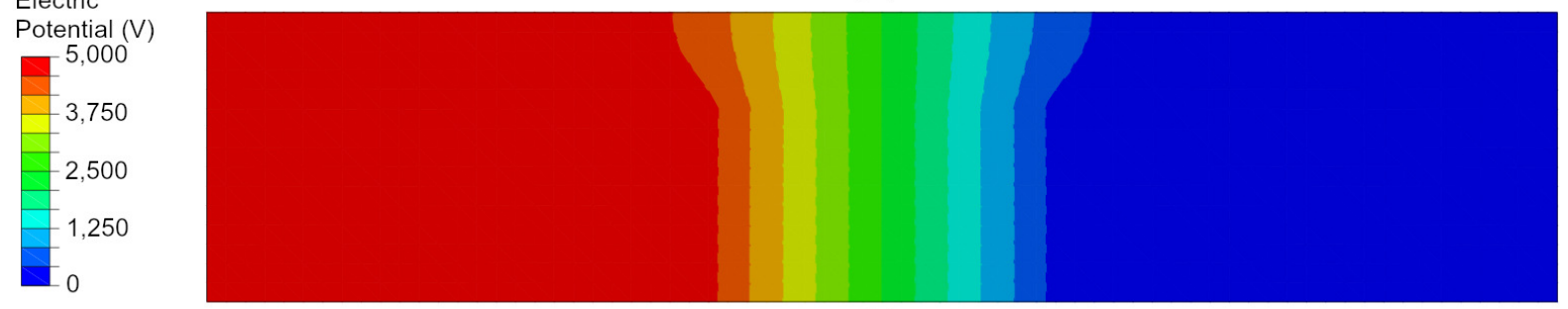

(b)

Figure 6: Finite element simulation of ohmic heating in the treatment chamber after a PEF treatment of $2.5 \mathrm{kV} / \mathrm{cm} \mathrm{at} 50 \mathrm{~Hz}$. (a) Temperature field. (b) Electric potential. 


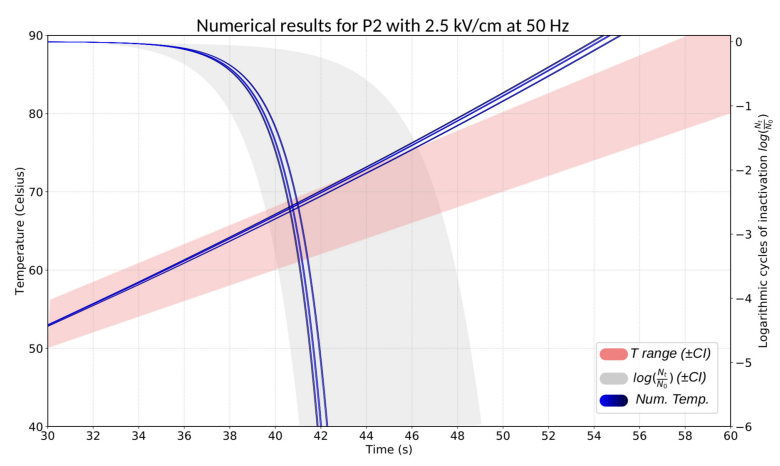

(a)

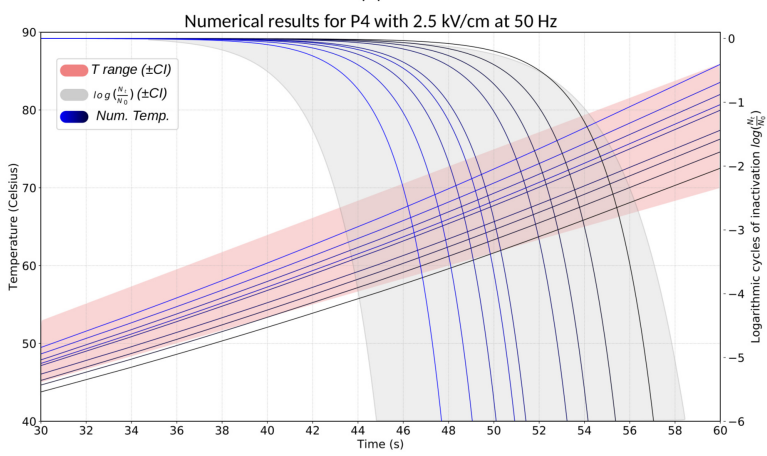

(c)

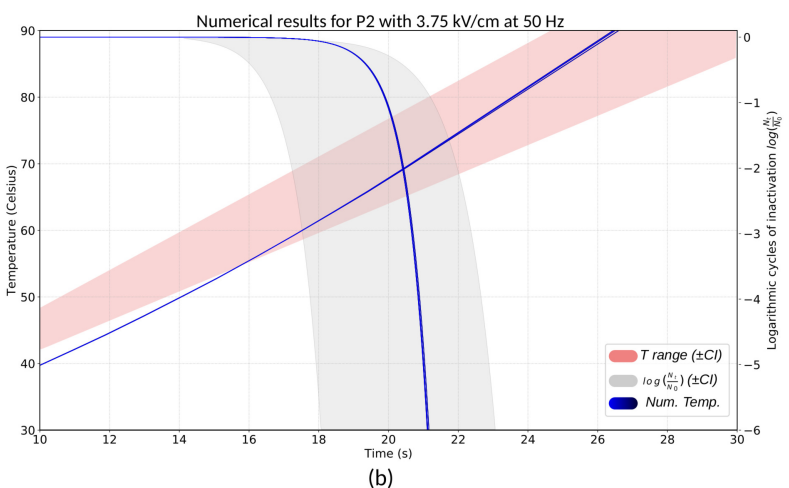

Numerical results for $\mathrm{P} 4$ with $3.75 \mathrm{kV} / \mathrm{cm}$ at $50 \mathrm{~Hz}$

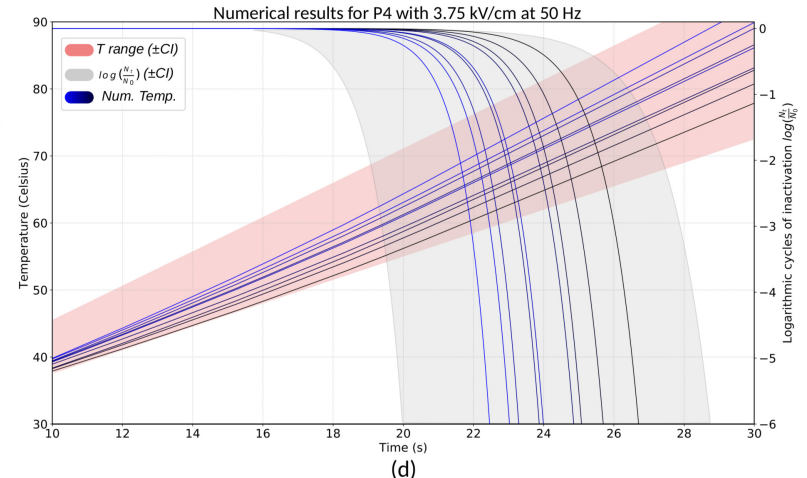

(d)

Figure 7: Simulated temperature and microbial inactivation (black-to-blue gradient lines) estimated by numerical simulation in positions P2 (a and c) and P4 (b and d) for simulations at $2.5 \mathrm{kV} / \mathrm{cm}$ and $50 \mathrm{~Hz}$ (a and b), and at $3.75 \mathrm{kV} / \mathrm{cm}$ and $50 \mathrm{~Hz}(\mathrm{c}$ and d). Shaded areas represent the experimental ranges shown in Figure 5 


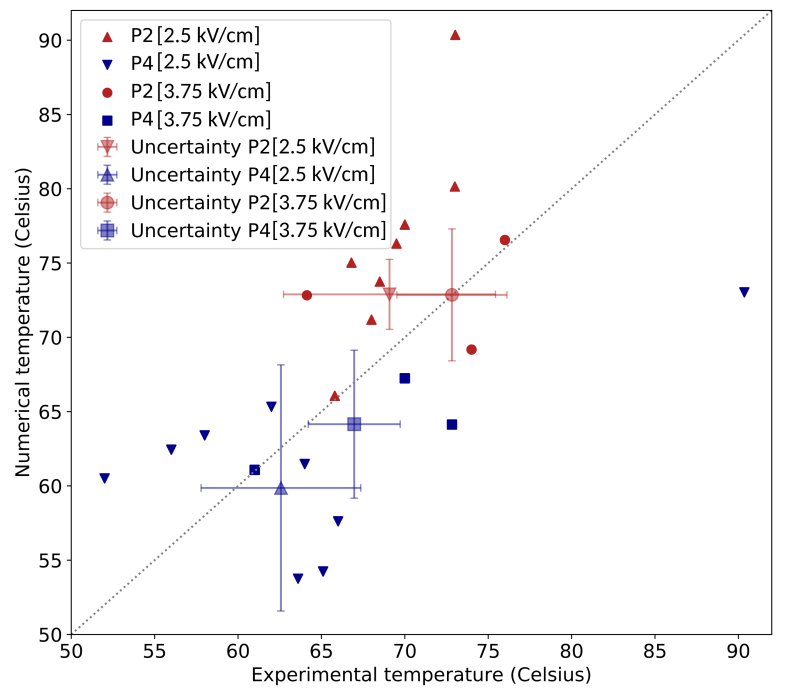

Figure 8: Correlation between experimental (x-axis) and simulated temperatures (y-axis). Triangles and inverted triangles represent measurements for experiments at $2.5 \mathrm{kV} / \mathrm{cm}$ and $50 \mathrm{~Hz}$; circles and squares represent measurements for $3.75 \mathrm{kV} / \mathrm{cm}$ and $50 \mathrm{~Hz}$, in red for position P2 (center) and in blue for position P4 (corner), respectively. Vertical error-bars represent uncertainty in experimental measurements, and horizontal error-bars represent uncertainty in numerical measurements (for experiments of different treatment time). 